\title{
Bilateral Stress Fracture of Patella during Jogging. A Brazilian Case Report
}

\section{Lourenço Galizia Heitzmann ${ }^{1,2 *}$, Pedro Bragato Romanholi ${ }^{1}$, Paulo Afonso Lages Gonçalves Filho ${ }^{1}$, Rogerio Teixeira de Carvalho ${ }^{1}$, Edu- ardo Angoti Magri ${ }^{1}$, Raphael Battisti ${ }^{1}$, Nataly Cristina Reis Uzelin ${ }^{1}$, Erika Saori Gushiken ${ }^{1}$, Rolando Gustavo Toledo Pacheco ${ }^{1}$ and Mauro Freitas Daher Junior ${ }^{1}$}

${ }^{1}$ Orthopedics and Traumatology Department, Hospital do Servidor Público

Estadual de São Paulo (HSPE), São Paulo, SP, Brazil

${ }^{2}$ Postgraduate Program of the Instituto de Assistência Médica ao Servidor Público

Estadual (IAMSPE), São Paulo, SP, Brazil

*Corresponding Author: Lourenço Galizia Heitzmann, Orthopedics and Traumatology Department, Hospital do Servidor Público Estadual de São Paulo (HSPE) and Postgraduate Program of the Instituto de Assistência Médica ao Servidor Público Estadual (IAMSPE), São Paulo, SP, Brazil.
Received: July 27, 2021

Published: October 13, 2021

(C) All rights are reserved by Lourenço Galizia

Heitzmann., et al.

\begin{abstract}
Bilateral stress fracture of the patella is an extremely rare condition. It is related to sports overuse and repetitive trauma. Some metabolic and hormonal abnormalities are associated as risk factors.

This report presents a case of atraumatic bilateral patella fracture in a female amateur runner treated in our institution surgically. The affected patient recovered function completely, returning to sport activity in a few months.
\end{abstract}

Keywords: Fractures Bone; Fractures Stress; Patella

\section{Introduction}

Stress fracture of the patella was described for the first time in English by Devas in 1960 [1]. This specific fracture is characterized by the failure of bone integrity caused by cyclic tensile forces through the patella, without history of acute or previous trauma.

It is a rare condition that affects people specially during the second to fourth decade of life. Patellar tendinopathy occurs frequently before this kind of fracture [2].

The first case of generic stress fracture mentioned in the literature was by Breithaupt in 1855 [3]. But recently during the $20^{\text {th }}$ century, these fractures were observed in military personnel mentioned in 1943 by Muller and in athletes during the next decades [4].

The first reported bilateral patellar fracture case was in 1956 [5]. Another case of bilateral lower pole patellar fracture was reported in 1981, affecting a basketball player [6]. Another case was described in 1983, relating the same kind of lesion found in another basketball player. A single case of a 64-year-old long-distance runner has been reported in the medical literature $[7,8]$.

The etiology of this type of fracture is unknown, however multiple risk factors have been discussed. These factors include bio- 
mechanical abnormalities, nutritional imbalance, and metabolic/ hormonal dysfunctions (especially in women). The female athlete triad including exercise overload, hormonal disturbance and osteopenia is a syndrome closely related to stress fracture, probably by sharing risk factors $[9,10]$.

Stress fractures occur most commonly in the lower limbs during physical exercises like training or competing, especially in running and jumping sports. They are related to sporting gestures (athlete dexterity, body mechanics and typical movements related to the activity), overload and overtraining [11,12]. Persistent anterior pain in the knees should be investigated as they increase suspicion about a possible patellar fracture prodrome in athletes [13].

Patellar stress fracture usually occurs during sport practice. It causes anterior knee pain, local edema and usually inability to keep stand position or extend the affected knee. There is usually a report of a click right before the onset of these symptoms [13].

There are several classifications for stress fracture, but most are difficult to apply. The Kaeding-Miller classification system incorporates clinically relevant parameters and is easily used for all stress fractures. It presents intra-observer and inter-observer reliability [14].

The fracture may be incomplete or complete, with or without impairment of knee extension function. Incomplete fractures usually are treat non-surgically using a plastered immobilization and rest until obtaining bone heal [13].

Complete fractures that compromise knee function should be treated surgically through bone osteosynthesis and/or tendon repair [11-13].

\section{Case Report}

A female 51-years-old amateur street runner came to our Orthopedic Emergency presenting intense anterior knee pain associated with inability to stay stand or walk after training for a competition. The patient has been training the last three years, mean load of 29 miles per week.

She had previous history of anterior knee pain during the last six months, being diagnosed with patellar chondromalacia after clinical examination, without any image study. No other associated condition found by that time.
The patient had no major clinical comorbidities. She was not in use of any medication or recreational drugs. Recent laboratory tests showed a vitamin D dosage of $22 \mathrm{ng} / \mathrm{ml}$. DXA densitometry testing accused $0.92 \mathrm{~g} / \mathrm{cm}^{2}$ in L4 vertebral body meaning osteopenia for her age.

During the training, she spontaneously felt a severe pain and cracking in the right knee followed by the same symptoms in the left knee. She became unable to keep stand position and felt to the ground. The patient affirms she had no direct trauma to the knee during the fall.

The physical findings included discrete local edema and inability to extend the knees actively. A patellar gap was palpated bilaterally. Simple radiographs revealed a transverse pattern fracture at the middle portion of both patella with a displacement greater than five millimeters (Figure 1).

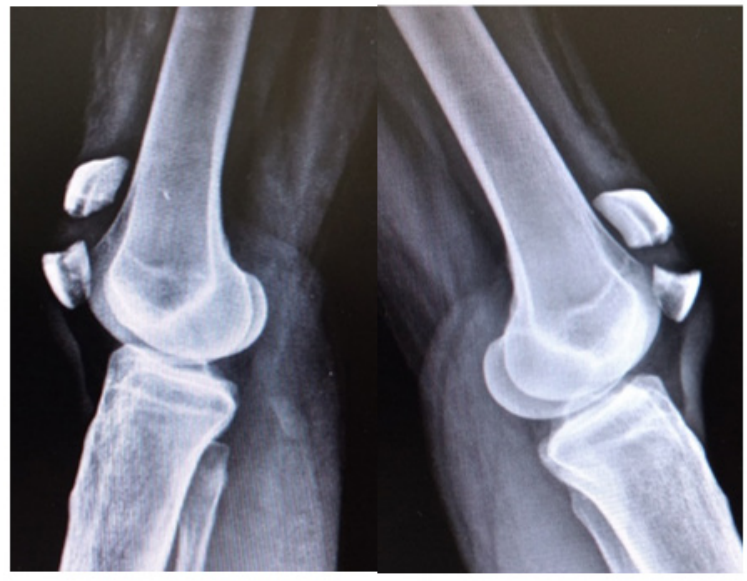

Figure 1: Lateral radiographs of both knees showing a transverse pattern patellar fracture in a 51 years-old amateur runner - the patient of this case report.

The patient was hospitalized for surgical treatment of this condition due to displacement of the fracture, bilateral commitment, and loss of knee extension function.

An anterior median incision was performed over the patella location, followed by dissection of tissues to reach the fractured 
bone. Patellar tendon and retinaculum tears were observed. No lesion of the patellofemoral cartilage was found. Patella fixation was performed using an "eight-pattern" steel wire tension band passed through two parallel $8 \mathrm{~mm}$ cannulated screws (Figure 2). We did not collect any tissue for histological analysis.

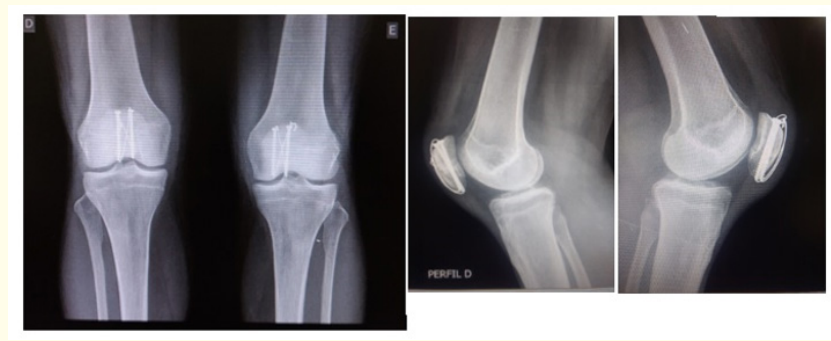

Figure 2: Surgical treatment of bilateral patellar stress fracture using cannulated screws tension band - the patient of this case report.

In the postoperative period, the patient complained about mild to moderate pain at the surgery site, managed with usual analgesic drugs. She was held hospitalized for two days to perform passive range- of- motion exercises and to receive physiotherapy team orientations.

During the next four weeks, the patient maintained the exercises without standing or walking. Then walking was reintroduced progressively, respecting tissue healing. Following the next step, gait rehabilitation and progressive resistance exercises for muscle strengthening were introduced.

Three months after surgery, the patient was fully recovered with normal gait, full range-of-motion in both knees, no local pain or edema.

\section{Discussion}

Patella stress fracture is a rare condition. Literature reunites a few dozens of cases throughout the years. Since the first publication in English, 39 cases in total to date have been documented. Thirty-five cases are tabulated in Brown., et al. and a few additional cases reports are noted in other texts [15].
The most common affected sites of stress fracture in adult athletes are tibia, fibula, metatarsal and navicular bones. Patella stress fracture is a rare condition, especially those with bilateral lesions.

There are two types of stress fractures: fatigue and insufficiency. In fatigue fracture, an incomplete or complete fracture occurs in a healthy bone submitted to cyclic stress through repetitive movements. In fractures due to insufficiency, the bone presents with osteopenia or osteoporosis leading to a fracture even under a physiologic load [15].

Running applies ground reaction force over the limbs five times greater than walking. The result of the excess tension force leads to an accumulation microtrauma that can lead to bone reaction and predisposition to failure [11,16-19].

Among the intrinsic risk factors, the decrease in bone mass is the main feature related to stress fractures [10]. An association between a family history of osteoporosis in first-degree relatives increases the risk of developing a stress fracture among athletes up to three times [16]. Low intakes of vitamin D and calcium as well as iron deficiency anemia are risk factors, hormonal disturbances like abnormal menstrual cycle also are related to detriment of bone quality $[9,10]$.

However, more study is needed to establish evidence-based associations between stress fracture and age, calcium intake, and age at menarche [15].

Our patient presented the history of running practice for the last three years from the incident, low serum dosage of vitamin D and low bone mass (osteopenia) that may have contributed to the onset of the stress fracture.

Radiography remains the initial imaging examination in a patient with suspected fracture stress. Several options are available for further evaluations, depending on the stage of the injury, but most experts agree that MRI is the gold standard because of its high sensitivity and specificity both for the early or late diagnosis $[11,13]$. MRI can show bone disturbances before any radiographic changes become evident [13].

According to X-Ray images and clinical aspects, our patient presented a stage IV Kaaeding-Miller stress fracture (evident displaced patella fracture) [14]. 


\section{Conclusion}

Bilateral stress fracture of the patella is a very rare condition and it is probably related to bone fatigue. Bilateral lesions are harder to find.

In this reported case, the surgical treatment was indicated due to the type of fracture, bilateral lesion, loss of extensor function and patient demand as athlete.

The treatment was successful, and the patient recovered functionality and returned to sports practice.

\section{Bibliography}

1. Devas MB. "Stress fractures of the patella". The Journal of Bone and Joint Surgery British 42B (1960): 71-74.

2. Teitz CC and Harrington RM. "Patellar stress fracture". American Journal of Sports Medicine 20.6 (1992): 761-765.

3. Breithaupt MD. "Zur Pathologie des menschlichen fusses". Medizin Zeitung 24 (1855): 196.

4. Cosman F., et al. "Determinants of stress fracture risk in United States Military Academy cadets". Bone 55.2 (2013): 359-366.

5. Korzh AA. "Bilateral fracture of patella". Vestnik Khirurgii Imeni 77.2 (1956): 121-123.

6. Tibone JE and Lombardo SJ. "Bilateral fractures of the inferior poles of the patellae in a basketball player". American Journal of Sports Medicine 9.4 (1981): 215-216.

7. Hensal F., et al. "Bilateral patellar fractures from indirect trauma. A case report". Clinical Orthopaedics and Related Research 178 (1983): 207-209.

8. Carneiro M., et al. "Bilateral stress fracture of the patellae: a case report”. Knee 13.2 (2006): 164-166.

9. Gordon CM and LeBoff MS. "The Female Athlete Triad: A Clinical Guide (book)". Springer, New York (2015).

10. Kelsey JL., et al. "Risk factors for stress fracture among young female cross-country runners". Medicine and Science in Sports and Exercise 39.9 (2007): 1457-1463.
11. Drabicki RR., et al. "Stress fractures around the knee". Clinic in Sports Medicine 25.1 (2006): 105-115.

12. Garcia-Mata S., et al. "Transverse stress fracture of the patella: a case report". Clinical Journal of Sport Medicine 6.4 (1996): 259-261.

13. Orava S., et al. "Diagnosis and treatment of stress fracture of the patella in athletes". Knee Surgery, Sports Traumatology, Arthroscopy 4.4 (1996): 206-211.

14. Kaeding CC and Miller TL. "The comprehensive description of stress fractures: a new classification system". Journal of Bone and Joint Surgery American 95 (2013): 1214-1220.

15. Knight JE and Brown G. "Longitudinal Patella Stress Fracture in an Ultra-Marathon Runner". Annals of Sports Medicine and Research 4.2 (2017): 1104.

16. Loud KJ., et al. "Family history predicts stress fracture in active female adolescents”. Pediatrics 120.2 (2007): 364-372.

17. Mason RW., et al. "Patellar fatigue fractures". Skeletal Radiology 25.4 (1996): 329-332.

18. Nalla RK., et al. "Letters: mechanistic fracture criteria for the failure of human cortical bone". Nature Materials 2 (2003): 164-168.

19. Behrens SB., et al. "Stress fractures of the pelvis and legs in athletes: a review". Sports Health 5 (2013): 165-174.

\section{Volume 4 Issue 11 November 2021 (c) All rights are reserved by Lourenço Galizia Heitzmann.,} et al. 